\title{
Slowly eroding lesions in multiple sclerosis
}

\author{
Varun Sethi, Govind Nair, Martina Absinta, Pascal Sati, Arun Venkataraman, Joan Ohayon, \\ Tianxia Wu, Kelly Yang, Colin Shea, Blake E Dewey, Irene CM Cortese and Daniel S Reich
}

\section{Abstract}

Background: At autopsy, $20 \%-40 \%$ of chronic multiple sclerosis (MS) lesions are labeled "slowly expanding" and feature myelin phagocytosis at the lesion edge. As pathological lesion classification relies on a single, terminal time point, the rate of lesion expansion cannot be directly measured.

Objective: To study long-term volume changes in individual MS lesions.

Methods: Volumes of individual lesions on proton density magnetic resonance imaging (MRI) acquired between 1992 and 2015 were measured in 22 individuals (one lesion per person). After correction for acquisition protocol, a mixed model evaluated lesion volume changes.

Results: The mean (standard deviation) lesion volume at baseline was $142(82) \mathrm{mL}$, falling to $74(51) \mathrm{mL}$ after 16 (3) years. All lesions shrank over time. Change in lesion volume did not correlate with change in supratentorial brain volume $(p=0.33)$. In simulations, the results could be explained by a process of slow radial expansion superimposed on substantially more rapid resorption of damaged tissue.

Conclusion: We noted sustained radiological contraction of MS lesions, a surprising result given that fresh myelin breakdown products within chronic active lesions are observed relatively frequently at autopsy. Therefore, the primary pathological process in chronic lesions, even those described as "slowly expanding," is likely to be tissue loss.

Keywords: Slowly expanding lesion, multiple sclerosis, magnetic resonance imaging, proton density

Date received: 14 March 2016; revised: 16 May 2016; accepted: 24 May 2016

\section{Introduction}

Multiple sclerosis (MS) is an inflammatory disease of the central nervous system characterized by demyelination within focal "lesions." In the white matter, lesions form around veins and may show partial loss of axons, variable degrees of reactive astrocytic scar formation, inflammation, demyelination, and remyelination - all of which may coexist within the same lesion. Based on the degree and nature of the immune reaction observed at autopsy, white matter lesions (WML) have been classified as "classically active," "slowly expanding" (SEL) or "smoldering," 2 and "chronic inactive." 3 At autopsy, particularly in progressive MS, approximately $20 \%{ }^{2}-40 \%^{3}$ of WML are categorized as SEL, characterized by a low degree of inflammation and microglial activation at the lesion edge. ${ }^{3}$ In these lesions, ongoing myelin destruction has been hypothesized to contribute to accrual of disability in progressive MS. ${ }^{4}$ However, as pathological lesion classification relies on analysis of a single time point, the rate of "expansion" over time - or even whether
SEL truly enlarge in the first place-remains speculative at best.

Magnetic resonance imaging (MRI) with proton density (PD) weighting has long been to study MS lesions $^{5-7}$ at a variety of magnetic field strengths. ${ }^{8,9} \mathrm{PD}$ images, though helpful for detecting lesions, are not thought to report specifically on the pathological changes of demyelination, remyelination, inflammation, and edema. ${ }^{10}$ Therefore, it remains exceedingly difficult to assess the pathological status of an individual lesion based on scans taken at a single time point. In the search for alternative noninvasive surrogates, lesion evolution over time is particularly attractive, as the pathological classification scheme suggests distinct patterns in this domain. In principle, by nature of their pathology, classically active lesions might be expected to change rapidly, SEL to slowly enlarge over time, and chronic inactive lesions to shrink as tissue is lost. Identifying the relative proportions of lesions of these various types could in principle be
Multiple Sclerosis Journal

$1-9$

DOI: $10.1177 /$

1352458516655403

(C) The Author(s), 2016.

Reprints and permissions:

http://www.sagepub.co.uk/

journalsPermissions.nav

Correspondence to: DS Reich

Translational Neuroradiology Section, National Institute of Neurological Disorders and Stroke (NINDS), National

Institutes of Health (NIH),

10 Center Drive MSC 1400 Building 10 Room 5C103, Bethesda, MD 20852, USA reichds@ninds.nih.gov

Varun Sethi

Govind Nair

Martina Absinta Pascal Sati

Arun Venkataraman

Joan Ohayon

Tianxia Wu

Kelly Yang

Colin Shea

Blake E Dewey

Irene CM Cortese

Daniel S Reich

National Institute of

Neurological Disorders and

Stroke (NINDS), National

Institutes of Health (NIH)

Bethesda, MD, USA 
Table 1. Summary of proton density acquisition protocols.

\begin{tabular}{|c|c|c|c|c|}
\hline Sequence type & SE (VEMP) & FSE & TSE/FSE & FSE \\
\hline Scanner manufacturer & GE & GE & $\begin{array}{l}\text { SIEMENS/PHILIPS/ } \\
\text { GE }\end{array}$ & GE \\
\hline Field strength $(\mathrm{T})$ & 1.5 & 1.5 & 3 & 3 \\
\hline Receive coil & 8CH HEAD & 8CH HEAD & $\begin{array}{l}32 \mathrm{CH} \mathrm{HEAD} / 8 \mathrm{CH} \\
\mathrm{HEAD} / 8 \mathrm{CH} \mathrm{HEAD}\end{array}$ & 8CH HEAD \\
\hline In-plane resolution & $1 \mathrm{~mm} \times 1 \mathrm{~mm}$ & $1 \mathrm{~mm} \times 1 \mathrm{~mm}$ & $1 \mathrm{~mm} \times 1 \mathrm{~mm}$ & $0.5 \mathrm{~mm} \times 0.5 \mathrm{~mm}$ \\
\hline Slice thickness (mm) & $5 \mathrm{~mm}$ & $3 \mathrm{~mm}$ & $3 \mathrm{~mm}$ & $3 \mathrm{~mm}$ \\
\hline Number of slices & 27 & 42 & $54 / 55 / 50$ & 52 \\
\hline $\mathrm{TR}(\mathrm{ms})$ & 2000 & 3400 & $3000 / 3500 / 5700$ & 5300 \\
\hline $\mathrm{TE}(\mathrm{ms})$ & 20 & $11-12$ & $11 / 15 / 12$ & 23 \\
\hline Flip angle $\left(^{\circ}\right)$ & 90 & 90 & $150 / 90 / 90$ & 90 \\
\hline $\begin{array}{l}\text { Number of scans with } \\
\text { these specifications }\end{array}$ & 107 & \multicolumn{2}{|c|}{ Total at $1 \times 1 \times 3=150$} & 5 \\
\hline \multicolumn{5}{|c|}{$\begin{array}{l}\text { CH: channel; SE: spin echo; VEMP: variable echo multiplanar; FSE: fast spin echo; TSE: turbo spin echo; TR: repetition time; TE } \\
\text { echo time. } \\
\text { Two scans were acquired at } 0.3 \times 0.3 \times 0.3 \text {, four scans at } 1 \times 1 \times 1 \text {, and one scan at } 0.5 \times 0.5 \times 0.5 \text {. }\end{array}$} \\
\hline
\end{tabular}

used to improve our understanding of the relationship between imaging abnormalities and clinical disability in MS, which is at present less than satisfactory. ${ }^{11-14}$ Furthermore, such an approach might identify specific lesions that could be targeted in trials of new therapies designed to protect tissue at risk or promote its repair. In this study, we characterized the evolution of individual chronic WML by investigating long-term changes (15-20 years) in lesion volume (LV) using PD MRI. We selected this imaging contrast for the primary analysis as it has been used for many years in MS MRI research to study these lesions and therefore allows an opportunity for long-term study.

\section{Methods}

\section{Participants and lesions}

We selected for study people with multiple sclerosis (PwMS) who had been seen in our clinic and had archived MRI scans over the course of approximately two decades. A total of 22 PwMS, whose first scan was between 1992 and 1999, were studied based on the identification of at least one discrete, non-enhancing, supratentorial WML at baseline, which remained discrete for the duration of the follow-up. Scans were obtained following informed consent under institutional review board-approved clinical research protocols.

\section{Data acquisition}

MRI scans, including (variably) PD, T1, T2, and T2-fluid-attenuated inversion recovery (FLAIR), were acquired between 1992 and 2015, using a variety of protocols and slice thicknesses, on either a $1.5 \mathrm{~T}$ (GE) or a $3 \mathrm{~T}$ (Siemens Skyra, Philips Achieva, or GE HDx) scanner (Table 1). Visually, the PD images had the most consistent contrast over time. A total of 269 scans were collected with in-plane resolution of $1 \mathrm{~mm} \times 1 \mathrm{~mm}$, $0.5 \mathrm{~mm} \times 0.5 \mathrm{~mm}$, or $0.3 \mathrm{~mm} \times 0.3 \mathrm{~mm}$. The slice thickness was $5 \mathrm{~mm}(1992-2002 ; n=107), 3 \mathrm{~mm}(2003$ $2015 ; n=155), 1 \mathrm{~mm}(2014-2015 ; n=4)$; one scan had slice thickness of $0.5 \mathrm{~mm}$ and two scans had slice thickness of $0.3 \mathrm{~mm}$. Clinicians recorded detailed medical histories and neurological examinations, including estimations of the Expanded Disability Status Scale (EDSS), periodically throughout the study period. In addition to demographic details, disease phenotype and history of disease-modifying therapy (DMT) and treatment with steroids were also recorded.

To study the effect of slice thickness on estimation of LV, PD scans (in-plane resolution $1 \mathrm{~mm} \times 1 \mathrm{~mm}$ and slice thickness of 1,3 , or $5 \mathrm{~mm}$ ) were obtained at the same sitting from 15 PwMS on the 3T Skyra scanner using a routine dual-echo sequence.

\section{Image analysis}

Estimation of lesion and supratentorial volume. Lesions were marked on unregistered, unnormalized PD images at a median of 13 time points (range: 2-19) using the image analysis software JIM 7.0. A contour was drawn around the entirety of the lesion on every slice where it was visible, and the LV thus estimated was noted at each successive time 
Table 2. Lesion volume at baseline and last time point for all 22 participants.

\begin{tabular}{|c|c|c|c|c|c|c|c|c|c|}
\hline Lesion no. & $\begin{array}{l}\text { Age at } \\
\text { baseline } \\
\text { (years) }\end{array}$ & Sex & $\begin{array}{l}\text { Disease } \\
\text { stage at } \\
\text { baseline }\end{array}$ & $\begin{array}{l}\text { Disease } \\
\text { stage at } \\
\text { follow-up }\end{array}$ & $\begin{array}{l}\text { Unadjusted } \\
\text { LV at } \\
\text { baseline }(\mu \mathrm{L})\end{array}$ & $\begin{array}{l}\text { Unadjusted } \\
\mathrm{LV} \text { at last time } \\
\text { point }(\mu \mathrm{L})\end{array}$ & $\begin{array}{l}\text { No. of } \\
\text { scans } \\
\text { available }\end{array}$ & $\begin{array}{l}\text { Duration } \\
\text { of follow- } \\
\text { up (years) }\end{array}$ & $\begin{array}{l}\text { Annual \% change } \\
\text { from mixed- } \\
\text { effects model }\end{array}$ \\
\hline 1 & 34 & M & RRMS & RRMS & 156.7 & 81.6 & 8 & 17.4 & -4.33 \\
\hline 2 & 31 & M & & SPMS & 145.7 & 35 & 15 & 19.3 & -7.59 \\
\hline 3 & 30 & M & RRMS & SPMS & 153.3 & 87.3 & 13 & 21.5 & -2.00 \\
\hline 4 & 36 & $\mathrm{~F}$ & RRMS & SPMS & 83.4 & 6 & 13 & 15.8 & -9.23 \\
\hline 5 & 26 & $\mathrm{~F}$ & RRMS & SPMS & 144.4 & 55.6 & 2 & 16.7 & -5.80 \\
\hline 6 & 20 & M & RRMS & SPMS & 190 & 133.6 & 11 & 14.8 & -4.66 \\
\hline 7 & 28 & M & RRMS & RRMS & 129.5 & 68.9 & 19 & 19.4 & -2.49 \\
\hline 8 & 29 & $\mathrm{~F}$ & RRMS & RRMS & 54 & 20.6 & 14 & 13.8 & -4.57 \\
\hline 9 & 31 & M & RRMS & RRMS & 151.6 & 53 & 15 & 14.1 & -5.38 \\
\hline 10 & 34 & $\mathrm{~F}$ & RRMS & RRMS & 368 & 171 & 15 & 15.9 & -2.56 \\
\hline 11 & 38 & $\mathrm{~F}$ & RRMS & SPMS & 40.2 & 69.8 & 14 & 19.0 & -1.65 \\
\hline 12 & 49 & $\mathrm{~F}$ & RRMS & RRMS & 56.9 & 12.8 & 13 & 19.5 & -6.67 \\
\hline 13 & 14 & $\mathrm{~F}$ & RRMS & SPMS & 172.8 & 139.1 & 18 & 17.7 & -2.35 \\
\hline 14 & 39 & M & RRMS & RRMS & 291.8 & 204 & 15 & 18.9 & -1.77 \\
\hline 15 & 35 & $\mathrm{~F}$ & & SPMS & 211.1 & 54 & 13 & 15.8 & -10.30 \\
\hline 16 & 25 & $\mathrm{~F}$ & RRMS & RRMS & 85.3 & 39.6 & 17 & 17.3 & -4.12 \\
\hline 17 & 37 & $\mathrm{~F}$ & RRMS & SPMS & 92 & 102.5 & 16 & 16.9 & -2.27 \\
\hline 18 & 23 & M & RRMS & RRMS & 221.3 & 102 & 3 & 16.5 & -4.51 \\
\hline 19 & 47 & $\mathrm{~F}$ & RRMS & SPMS & 132.9 & 42.5 & 13 & 15.7 & -4.66 \\
\hline 20 & 27 & $\mathrm{~F}$ & RRMS & RRMS & 59.3 & 52.4 & 3 & 5.8 & -3.81 \\
\hline 21 & 43 & $\mathrm{~F}$ & RRMS & RRMS & 144.9 & 66.4 & 13 & 14.7 & -5.02 \\
\hline 22 & 17 & $\mathrm{~F}$ & RRMS & RRMS & 34.6 & 35.4 & 3 & 13.4 & -3.14 \\
\hline Mean & 32 & & & & 141.8 & 74.2 & $\begin{array}{l}\text { Median } \\
13\end{array}$ & 16.4 & -4.5 \\
\hline $\begin{array}{l}\text { Standard } \\
\text { deviation }\end{array}$ & 9 & & & & 82.1 & 50.7 & $\begin{array}{l}\text { Range } \\
2-19\end{array}$ & 3.2 & 2.3 \\
\hline
\end{tabular}

point. For 13 randomly selected lesions, LV was manually computed by two trained raters (V.S. and A.V.) blinded to demographics. Inter-rater variability for manual estimation of LV was assessed using the intraclass correlation coefficient. The supratentorial volume was estimated using lesionTOADS. ${ }^{15}$ As FLAIR scans were required for this estimation, data were available for only 90 time points (of a total of 269).

Volume-slice thickness effect. Data were acquired by collecting PD scans at different slice thicknesses on the same day in 15 PwMS (6 of whom were among the 22 PwMS whose lesions were studied longitudinally). These data were then modeled, using simple linear regression, to derive a "transformed" (slice-thicknessadjusted, log-transformed) LV at each time point.

Analysis of longitudinal volume data. Transformed LV data were analyzed, first by comparing baseline and last time point (Table 2), and second by modeling volume change over time through mixed-effects models. Such models have been used for retrospective longitudinal data analysis in MS, ${ }^{16}$ are efficient even when including data from participants with few or missing time points, and yield participant-specific intercepts and slopes. Subject- and population-level effects are naturally accounted for in these models, providing a comprehensive interpretation of the data. As in our prior work, ${ }^{16,17}$ we included age as a fixed effect (instead of time since baseline) and sex and treatment as covariates. The association between the estimated lesion-specific slopes and clinical variables was studied using Pearson's correlation coefficient. Mixed-effects models were also used to study the rate of change in supratentorial volume over time. Pearson's correlation was used to study the association between annual changes in lesion and supratentorial volume derived from the random-effects estimates. 


\section{Simulations}

To better understand our data, we undertook simulations based on hypothesized patterns of lesion expansion and shrinkage that could simultaneously be taking place within a lesion. Spherical lesions were simulated as enlarging either as a result of inflammation at the lesion edge, which could increase LV in proportion to either the radius or the volume of the lesion, or alternatively at a fixed rate over time. Lesion shrinkage either could be most aggressive at the lesion center, where tissue damage is thought to be most severe, ${ }^{18}$ or alternatively could occur in a fixed amount per unit time over the entire extent of the lesion. The simulation was investigated in an attempt to understand the rate of decrease in LV. We modeled the parameters taking into account both expansion at the lesion boundary (proportional to radius or volume of lesion or at a fixed rate over time; supplement equations (2)-(4)) and resorption (either as a function of $\mathrm{LV}$ or at a fixed rate over time; supplement equations (5) and (6)).

The net change in LV was modeled as the difference between the volume gained through the expansion process and the volume lost through the shrinkage process. Mathematical details are provided in the online supplementary material. The simulation results were compared to the observed rates of change in LV from the mixed-effects model. The simulations were not intended to provide realistic geometric models but to provide an overall context for interpretation of the longitudinal data.

\section{Statistical analysis}

Statistical analysis used SAS version 9.3. Distribution of residuals was checked with the Shapiro-Wilk test. Results are expressed as mean (standard deviation, SD) unless otherwise mentioned, and $p<0.05$ was considered to be statistically significant.

\section{Results}

\section{Descriptive statistics}

Data from 22 PwMS (14F/8M, mean age at baseline scan 32 (9) years) were analyzed. At baseline, 20 had relapsing-remitting multiple sclerosis (RRMS); at the terminal scan, 12 still had RRMS and 10 had secondary-progressive multiple sclerosis (SPMS). Disease subtype was not classified in the initial clinic notes in two cases. Over 16 (3) years (range: 13-22 years), the median EDSS changed from 2.0 to 4.5 across all participants (1.5-2.0 for participants with RRMS at the last time point and 2.5-6 for those with SPMS).
The volumes of 22 lesions were estimated using PD scans. When possible, scans prior to "baseline" were checked to assess lesions' enhancement history. One lesion enhanced 6 months prior to baseline and one enhanced 18 months prior to baseline. For three lesions, no preceding scan was available. For the others, no enhancement was detected on any of the prior scans. Representative images from one person are shown in Figure 1. Across the cohort, raw (uncorrected) LV decreased from 142 (82) to $74(51) \mu \mathrm{L}$, with an annualized change of -4.1 (3.3) $\mu \mathrm{L} /$ year (Table 2). Volume estimates in 19 of 22 lesions were smaller at follow-up than at baseline, one lesion was essentially stable, and two lesions had slightly larger LV at follow-up. In 13 lesions for which LV was estimated twice by each of two raters, the intraclass correlation coefficient was 0.96 within raters and 0.87 across raters.

\section{Effect of slice thickness on LV}

Volumes of 15 discrete, supratentorial WML were acquired at different slice thicknesses within a single imaging session. Measurements at different slice thicknesses (Figure 2) were used to establish, through linear regression, the following relationship between voxel and LV (in $\mu \mathrm{L})$

$$
\begin{aligned}
\text { adjusted } \mathrm{LV}= & \log _{10}(\mathrm{LV}+1)+ \\
& 0.1065(\text { voxel volume }-3)
\end{aligned}
$$

The adjusted LV was used for the mixed-effects modeling.

\section{Mixed-effects modeling of volume changes}

The residuals using raw data were not distributed normally (Shapiro-Wilk test using Pearson residuals $p<0.0001$ ), whereas the residuals based on log transformation followed a normal distribution (ShapiroWilk test using Pearson residuals $p=0.1562$ ); we thus used log-transformed data for the mixed-effects model.

Mean rate of change of adjusted LV was $-4.5 \%$ $(2.3 \%)$ (range: $-10.3 \%$ to $-1.6 \%$ ) (Figure $3(a)$ ). In this analysis, no LV showed a positive slope to suggest true enlargement. Lesion-specific rates of change showed no correlation with baseline $\operatorname{EDSS}(p=0.29)$, last available EDSS $(p=0.29)$, or change in EDSS $(p=0.71)$. Furthermore, there was no association between lesion-specific rates of change and baseline $(p=0.72)$ or final $(p=0.31) \mathrm{LV}$. Variables considered as covariates included sex $(p=0.16)$, history of 


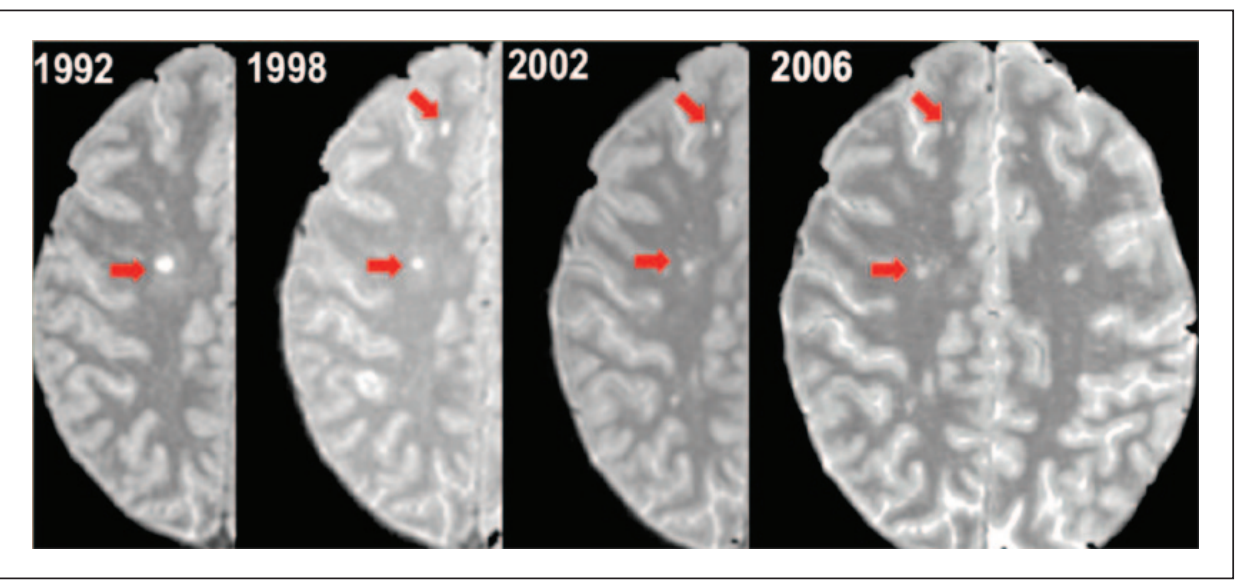

Figure 1. Proton density-weighted images acquired over 14 years in a representative individual with relapse-onset MS, showing a reduction in the size of two discrete white matter lesions (red arrows).

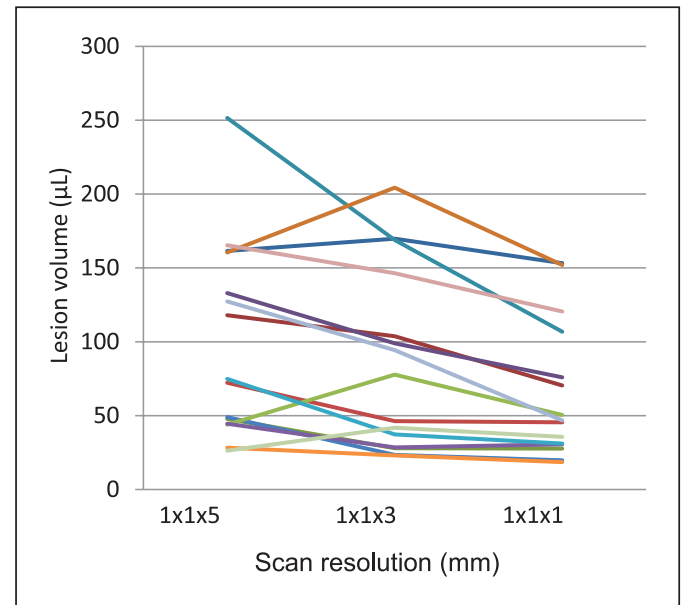

Figure 2. Scan resolution affects lesion volume. Each line represents a discrete lesion in a separate individual, all measured within a single scanning session.

treatment with DMT $(p=0.57)$, and treatment with steroids within 30 days prior to the scan $(p=0.14)$; these were dropped from the final model due to lack of association.

A similar model estimated the rate of change in untransformed supratentorial volume (Figure 3(b)). Across the population, supratentorial volume changed by a mean of -5 (2.6) (range: -10 to 1.5 ) $\mathrm{mL} / \mathrm{year}$. There was no correlation between the rates of change in lesion and supratentorial volume ( $p=0.33$; Figure $3(\mathrm{c})$ ).

\section{Simulation}

A series of simple simulations assessed the conditions under which a fixed rate of lesion shrinkage, of approximately $4.5 \%$ per year, could be realized. The simulations allowed simultaneous lesion expansion (at the edge, in proportion to either the radius or the volume of the lesion) and contraction (either at the center in proportion to the lesion radius, or homogeneously throughout the lesion). A constant proportional rate of lesion shrinkage was found to require models that assumed homogeneous tissue resorption throughout the lesion and was best recapitulated when expansion at the lesion edge was proportional to lesion radius. For example, using an initial lesion radius of $5 \mathrm{~mm}$, radial expansion of $1 \%$, and annual within-LV loss of $10 \%$, the model predicted a stable erosion rate of $\sim 4 \%$ per year (Figure 4 ; green line). On the contrary, a model that assumed tissue resorption only at the lesion center resulted in accelerating lesion erosion over time (Figure 4; red line), which was not reflected in our longitudinal data.

\section{Discussion}

The time-evolution of long-standing WML (one per person) was studied radiologically in 22 PwMS. After adjustment for scanning protocol and regression analysis, all lesions were found to shrink, at a mean rate of 4.5\% (SD: $2.3 \%$ ) per year, independent of sex or treatment with DMT or steroids. A mathematical simulation suggested that our findings may be explained by the overlap of multiple pathological processes, including tissue resorption within the lesion and inflammatory expansion at the lesion edge.

Chronic WML in MS can generally be grouped into inactive lesions (without ongoing inflammation) and "slowly expanding" or "smoldering" lesions (with peripheral inflammation and ongoing myelin phagocytosis)..$^{2,3}$ The suggestion of ongoing demyelination 


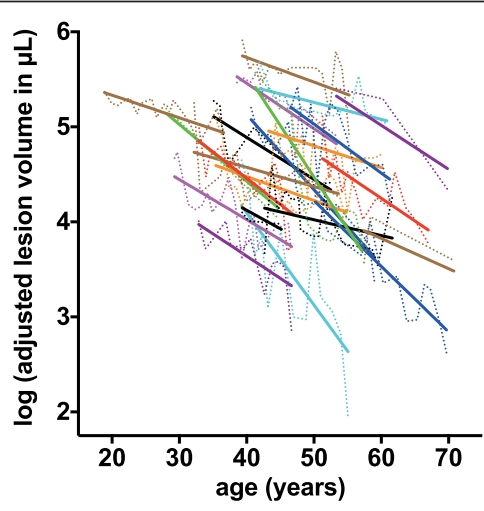

(a)

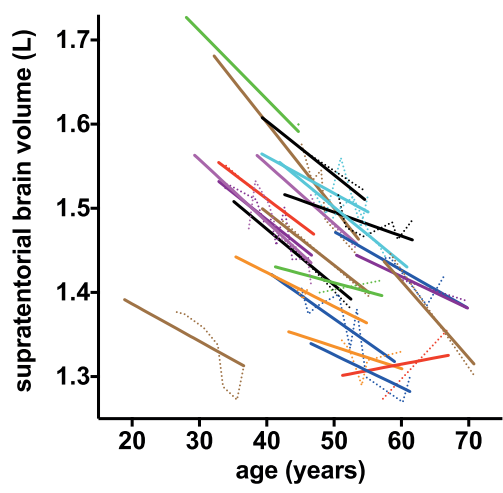

(b)

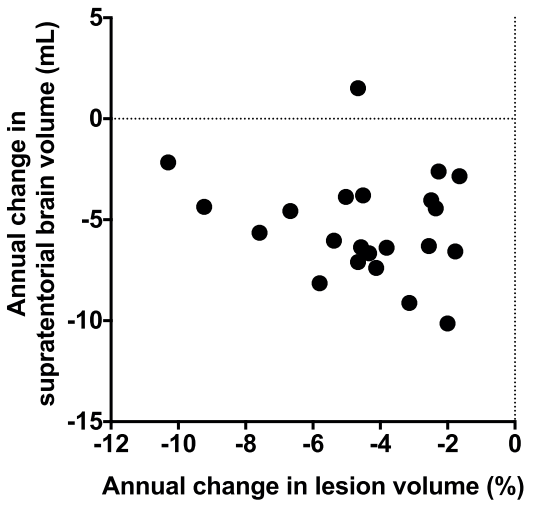

(c)

Figure 3. (a) The logarithm of the adjusted lesion volume (see equation (1)) tracked over time (dashed line) for all 22 lesions. The mixed-effects model fit for each lesion is also shown (solid lines). (b) Supratentorial brain volume tracked over time, derived from the subset of data that was suitable for volumetric analysis. The mixed-effects model fit for each lesion is also shown (solid lines). (c) The rate of change in lesion volume was uncorrelated with the rate of change in supratentorial brain volume.

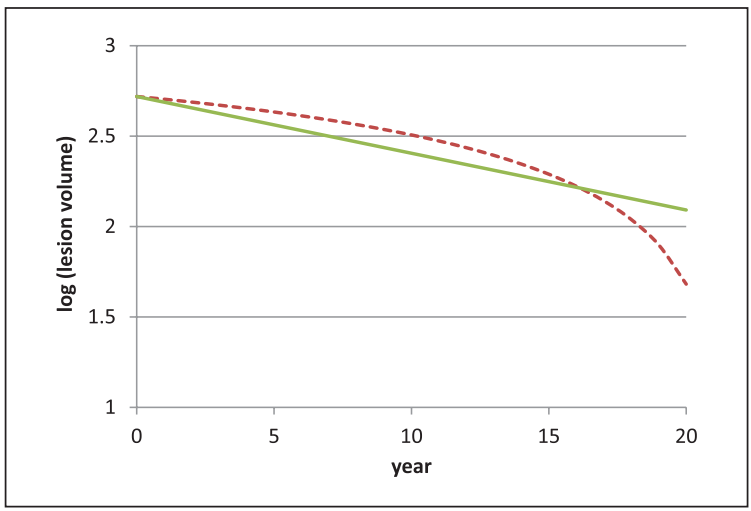

Figure 4. Simulations predict a stable erosion rate of $\sim 4 \%$ per year (solid green line) when assuming that lesion tissue is resorbed as a fixed percentage of the volume, even while the lesion undergoes expansion at the edge at a rate proportional to its radius. This finding is consistent with our longitudinal in vivo data. On the other hand (dashed red line), in the simulation assuming that tissue is preferentially resorbed at the lesion center, even with ongoing expansion at the edge, the rate of lesion erosion accelerates over time, a situation not recapitulated in vivo. has led to the concept that these lesions enlarge over time, ${ }^{19}$ though the rate of enlargement cannot be inferred directly from tissue-based analysis at autopsy. Enlarging lesions have also been reported in some earlier MRI studies ${ }^{20}$ and are commonly reported in clinical trials, but these are probably mostly lesions of recent onset.

The finding of sustained contraction in all the lesions that we imaged over a mean of 16 years was therefore surprising. One possible explanation for lesion contraction could be ongoing repair, such as remyelination. However, most remyelination is thought to occur within the first few months after lesion onset, perhaps up to 1 year, ${ }^{19,21-23}$ and the lesions studied herein were non-enhancing (i.e. chronic) even at the first time point. In our view, it is therefore more likely that the lesion contraction we observed was primarily the result of slow loss of damaged tissue, which must have overwhelmed any concurrent lesion enlargement. Indeed, our simulations demonstrated that reasonable rates of expansion and atrophy could generate 
the roughly constant proportional loss of LV that we observed. Of course, in the absence of tissue-based analysis, it is impossible to say whether any of the lesions in this study would have met the pathological definition of SEL. A model with only resorption might fit the data as well, if appropriate parameters for the resorption process are chosen. The simulations suggest that a slow expansion process could coexist with the resorption process, and as such our major conclusion is that whatever expansion is taking place in "slowly expanding lesions" could be more than counterbalanced by tissue loss.

Although expansion of chronic lesions into adjacent myelinated white matter and loss of axons traversing the same lesions can both, in principle, damage normal brain tissue, we found no correlation between rates of change in supratentorial brain volume and LV. Given that total white matter (including areas with both normal and abnormal signal on MRI) has been shown to be at most only slightly atrophic in MS, ${ }^{24}$ and not to change substantially over moderately long periods of time, ${ }^{16}$ our results seem to suggest that slow erosion of existing lesions does not, on its own, contribute substantially to tissue loss. However, we have considered only one lesion per person, and it is possible that a more comprehensive analysis is required to detect an effect of lesion erosion on brain atrophy. It should also be noted that axonal degeneration within individual lesions can have widespread effects, ${ }^{11,12,25-28}$ and these effects may depend on local connectivity patterns, thus potentially amplifying the erosive effect of withinlesion tissue loss. Detailed analysis of these effects is beyond the scope of this study and would be difficult to perform reliably with available data. It is also possible that the reduction in LV observed in our data may result from global degenerative processes, rather than the other way around. ${ }^{29,30}$

Clinical trials frequently report the number of new and enlarging lesions as measures of therapeutic efficacy $^{31,32}$ but provide limited information about longterm benefits of treatment. Interestingly, we found no consistent effect of sex and treatment with DMT or steroids on rates of LV change. Nonetheless, previous studies investigating lesion recovery patterns report a limitation of tissue damage and enhanced recovery under some treatments. 33,34 Further research can assess whether MRI outcomes, such as the long-term rate of LV change, may be useful to assess the effects of treatment on disease progression.

A limitation of our study is that by choosing lesions that remained discrete over nearly two decades, our data are subject to selection bias, meaning that we may have artificially excluded the class of SEL. Put another way, truly enlarging lesions are more likely to become confluent over time, particularly in people with high lesion load. To partially address this possibility, we analyzed four additional lesions that started discrete but became confluent during the course of the follow-up; none of those lesions was found to decrease in size prior to the point of becoming confluent (data not shown). Additional work, perhaps based on detailed lesion morphology assessed at ultra-highfield (7T) MRI, or even pathology, in comparison to historical MRI datasets, would be necessary to obtain a more complete understanding of the significance of LV changes over time.

In our modeling, we found that a fixed relative (percentage) change was more consistent with the observed data than an absolute change (comparative modeling not shown). It is possible that more complex nonlinear models, perhaps including inflection points related to treatment with immunomodulation and steroids, would be more accurate, but as our population was heterogeneous, and our dataset relatively small, we did not consider such scenarios here.

Another challenge of the present analysis is the heterogeneous and even subtle changes in MRI protocol parameters inevitable in long-term studies. In order to control for, to the extent possible, the effects of variability in protocol parameters, we chose to analyze fastspin-echo PD scans. We found that other than field strength and receiver coil type, the major change in the parameters of the PD scans over time was slice thickness, and its effect on computation of LV was modeled by scanning patients with several different slice thicknesses in a single sitting. We then mitigated the slice thickness effect by transforming all scans to a single slice thickness and using the resulting transformed data for all further analysis. We found that there was an overestimation in LV when lesions were imaged with thicker slices, which can be attributed to increased partial volume effects along the slice direction. ${ }^{35}$ We acknowledge, however, that our attempts to homogenize the data are fundamentally imperfect in the absence of a standard such as a realistic phantom scanned with the same techniques over the duration of follow-up.

Overall, our findings suggest that pathological descriptions based on single-time-point observations, such as the concept of SEL, may fail to correlate with in vivo findings, as assessed by MRI. In a broader sense, combining longitudinal LV information of the type reported here with detailed analysis of lesion imaging characteristics might provide useful information for participant selection in clinical trials, while 
simultaneously allowing improved understanding of the clinical impact of lesions on morbidity and progression of disease.

\section{Acknowledgements}

The authors thank Rita Devine and the NINDS Summer Internship Program, John Ostuni, and Roger Stone. They also thank the study participants, the Neuroimmunology Clinic for recruiting and evaluating the study participants and for coordinating the scans, and the NIH Functional Magnetic Resonance Imaging Facility.

\section{Declaration of Conflicting Interests}

The author(s) declared no potential conflicts of interest with respect to the research, authorship, and/or publication of this article.

\section{Funding}

The author(s) disclosed receipt of the following financial support for the research, authorship, and/or publication of this article: This study was supported by the Intramural Research Program of the National Institute of Neurological Disorders and Stroke.

\section{References}

1. Dawson J. The histology of disseminated sclerosis. $T$ Roy Soc Edin 1916; 50: 517-740.

2. Frischer JM, Weigand SD, Guo Y, et al. Clinical and pathological insights into the dynamic nature of the white matter multiple sclerosis plaque. Ann Neurol 2015; 78(5): 710-721.

3. Lassmann H. The pathologic substrate of magnetic resonance alterations in multiple sclerosis. Neuroimaging Clin N Am 2008; 18(4): 563-576.

4. Prineas JW, Kwon EE, Cho ES, et al. Immunopathology of secondary-progressive multiple sclerosis. Ann Neurol 2001; 50(5): 646-657.

5. Yousry TA, Seelos K, Mayer M, et al. Characteristic MR lesion pattern and correlation of T1 and T2 lesion volume with neurologic and neuropsychological findings in cerebral autosomal dominant arteriopathy with subcortical infarcts and leukoencephalopathy (CADASIL). AJNR Am J Neuroradiol 1999; 20: 91-100.

6. Yousry TA, Filippi M, Becker C, et al. Comparison of MR pulse sequences in the detection of multiple sclerosis lesions. AJNR Am J Neuroradiol 1997; 18(5): 959-963.

7. Gawne-Cain ML, O'Riordan JI, Thompson AJ, et al. Multiple sclerosis lesion detect in the brain: A comparison of fast fluid-attenuated inversion recovery and conventional T2-weighted dual spin echo. Neurology 1997; 49(2): 364-370.

8. Kollia K, Maderwald S, Putzki N, et al. First clinical study on ultra-high-field MR imaging in patients with multiple sclerosis: Comparison of $1.5 \mathrm{~T}$ and 7T. AJNR Am J Neuroradiol 2009; 30(4): 699-702.

9. Filippi M, Horsfield MA, Tofts PS, et al. Quantitative assessment of MRI lesion load in monitoring the evolution of multiple sclerosis. Brain 1995; 118(6): 1601-1612.

10. Pirko I and Johnson AJ. Neuroimaging of demyelination and remyelination models. In: Rodriguez M (ed.) Current topics in microbiology and immunology. Berlin: Springer, 2008, pp. 241-266.

11. Ciccarelli O, Brex PA, Thompson AJ, et al. Disability and lesion load in MS: A reassessment with MS functional composite score and 3D fast FLAIR. $J$ Neurol 2002; 249(1): 18-24.

12. Fisniku LK, Brex PA, Altmann DR, et al. Disability and T2 MRI lesions: A 20-year follow-up of patient with relapse onset of multiple sclerosis. Brain 2008; 131(3): 808-817.

13. Rinaldi F, Calabrese M, Grossi P, et al. Cortical lesions and cognitive impairment in multiple sclerosis. Neurol Sci 2010; 31(Suppl. 2): S235-S237.

14. Calabrese M, Poretto V, Favaretto A, et al. Cortical lesion load associates with progression of disability in multiple sclerosis. Brain 2012; 135(Pt 10): 29522961.

15. Shiee N, Bazin P-L, Ozturk A, et al. A topologypreserving approach to the segmentation of brain images with multiple sclerosis lesions. Neuroimage 2010; 49(2): 1524-1535.

16. Jones BC, Nair G, Shea CD, et al. Quantification of multiple-sclerosis-related brain atrophy in two heterogeneous MRI datasets using mixed-effects modeling. Neuroimage Clin 2013; 3: 171-179.

17. Borges IT, Shea CD, Ohayon J, et al. The effect of daclizumab on brain atrophy in relapsing-remitting multiple sclerosis. Mult Scler Relat Disord 2013; 2(2): 133-140.

18. Gaitán MI, de Alwis MP, Sati P, et al. Multiple sclerosis shrinks intralesional, and enlarges extralesional, brain parenchymal veins. Neurology 2013; 80(2): 145-151.

19. Lassmann H, Brück W and Lucchinetti CF. The immunopathology of multiple sclerosis: An overview. Brain Pathol 2007; 17(2): 210-218.

20. Tan IL, van Schijndel RA, Fazekas F, et al. Image registration and subtraction to detect active $\mathrm{T} 2$ lesions in MS: An interobserver study. J Neurol 2002; 249(6): 767-773. 
21. Prineas JW, Barnard RO, Kwon EE, et al. Multiple sclerosis: Remyelination of nascent lesions. Ann Neurol 1993; 33(2): 137-151.

22. Prineas JW and Parratt JDE. Oligodendrocytes and the early multiple sclerosis lesion. Ann Neurol 2012; 72(1): 18-31.

23. Wolswijk G. Chronic stage multiple sclerosis lesions contain a relatively quiescent population of oligodendrocyte precursor cells. J Neurosci 1998; 18(2): 601-609.

24. Shiee N, Bazin P-L, Zackowski KM, et al. Revisiting brain atrophy and its relationship to disability in multiple sclerosis. PLoS ONE 2012; 7(5): e37049.

25. Papadopoulou A, Müller-Lenke N, Naegelin Y, et al. Contribution of cortical and white matter lesions to cognitive impairment in multiple sclerosis. Mult Scler 2013; 19(10): 1290-1296.

26. Myhr KM, Riise T, Vedeler C, et al. Disability and prognosis in multiple sclerosis: Demographic and clinical variables important for the ability to walk and awarding of disability pension. Mult Scler 2001; 7(1): $59-65$.

27. Vrenken H, Seewann A, Knol DL, et al. Diffusely abnormal white matter in progressive multiple sclerosis: In vivo quantitative MR imaging characterization and comparison between disease types. AJNR Am J Neuroradiol 2010; 31(3): 541-548.

28. Seewann A, Vrenken H, Van der Valk P, et al. Diffusely abnormal white matter in chronic multiple sclerosis: Imaging and histopathologic analysis. Arch Neurol 2009; 66(5): 601-609.
29. Kezele IB, Arnold DL and Collins DL. Atrophy in white matter fiber tracts in multiple sclerosis is not dependent on tract length or local white matter lesions. Mult Scler 2008; 14(6): 779-785.

30. Spulber G, Niskanen E, Macdonald S, et al. Evolution of global and local grey matter atrophy on serial MRI scans during the progression from MCI to AD. Curr Alzheimer Res 2012; 9(4): 516-524.

31. Miller DH, Khan OA, Sheremata WA, et al. A controlled trial of natalizumab for relapsing multiple sclerosis. N Engl J Med 2003; 348(1): 15-23.

32. IFNB. Interferon beta- $1 b$ in the treatment of multiple sclerosis: Final outcome of the randomized controlled trial. The IFNB Multiple Sclerosis Study Group and The University of British Columbia MS/MRI Analysis Group. Neurology 1995; 45(7): $1277-1285$

33. Richert ND, Ostuni JL, Bash CN, et al. Interferon beta- $1 \mathrm{~b}$ and intravenous methylprednisolone promote lesion recovery in multiple sclerosis. Mult Scler 2001; 7(1): 49-58.

34. Bergh FT, Kümpfel T, Schumann E, et al. Monthly intravenous methylprednisolone in relapsingremitting multiple sclerosis-Reduction of enhancing lesions, T2 lesion volume and plasma prolactin concentrations. BMC Neurol 2006; 6(1): 19 .

35. Miller DH, Barkhof F, Frank JA, et al. Measurement of atrophy in multiple sclerosis: Pathological basis, methodological aspects and clinical relevance. Brain 2002; 125(8): 1676-1695.
Visit SAGE journals online http://msj.sagepub.com

\$SAGE journals 